\title{
Measurements of Balun and Gap Effects in a Dipole Antenna
}

\author{
Constantinos Votis ${ }^{1}$, Vasilis Christofilakis ${ }^{1,2}$, Panos Kostarakis ${ }^{1}$ \\ ${ }^{1}$ Physics Department, University of Ioannina, Panepistimioupolis, Ioannina, Greece \\ ${ }^{2}$ Siemens Enterprise Communications, Enterprise Products Development, Athens, Greece \\ E-mail:kvotis@grads.uoi.gr,basilios.christofilakis@siemens-enterprise.com,kostarakis@uoi.gr \\ Received February 12, 2010; revised March 15, 2010; accepted April 18, 2010
}

\begin{abstract}
In the present paper, design and analysis of a $2.4 \mathrm{GHz}$ printed dipole antenna for wireless communication applications are presented. Measurements on return loss and radiation pattern of this antenna configuration are included in this investigation. The printed dipole is combined with the feeding structure of a microstrip via-hole balun and is fabricated on an FR-4 printed-circuit-board substrate. Two inevitable discontinuities are introduced by this antenna architecture in the form of right-angle bends in the microstrip feed line and in the dipole's gap, respectively. The impact of mitering these bends in the reflection coefficient, resonance bandwidth and radiation pattern of antenna has been investigated by means of simulation and experiment.
\end{abstract}

Keywords: Printed Dipole, Integrated Balun, S-Parameters, Radiation Pattern

\section{Introduction}

The microstrip antenna architecture, in general, offers inherent narrow bandwidth and quite low gain. These limitations do not provide wide usage of these antennas in wireless applications systems. Besides, the evolution of wireless communications leads to more compact and small equipment that demanding antennas with smaller size and profile. Hence, the scientific community has started to investigate methods to improve this antenna architecture and provide better quality of services on wireless communication systems. In this way, many research activities were based on the printed dipole antenna because it has low profile, simple structure and omnidirectional radiation pattern. In order to develop this antenna configuration a printed dipole antenna with integrated balun and microstrip line as feeding structure was proposed [1-3]. Based on these considerations, we design and fabricate a $2.4-\mathrm{GHz}$ printed dipole antenna with integrated microstrip balun. This antenna design offers all the advantages of printed circuits and the corresponding geometry characteristics have been in detail studied and investigated [4-5]. In order to improve the bandwidth and the gain of this architecture we study and investigate the impact on variations of the $l$ and $w$ geometrical parameters on antenna performance. The first corresponds to the right angle bend in the microstrip balun and the second affects the dipole's arms. Details of the structure and design process are presented in the next section (Section 2 ). The corresponding simulated and measured results are presented and discussed in Section 3. The paper concludes in Section 4.

\section{Design Process and Structure}

The geometry and design parameters of the $2.4 \mathrm{GHz}$ printed dipole antenna are drawn in Figure 1. It is a modified antenna design that was introduced by the corresponding literature [1-4].

This printed dipole antenna was etched on Fr4 substrate with thickness $h=1.5 \mathrm{~mm}$ and permittivity $\varepsilon_{\mathrm{r}}=$ 4.4. The ground plane of the microstrip line and the dipole strips were printed at the bottom layer. A microstrip via-hole balun acts as an unbalance-to-balance transformer from the coaxial line to the printed dipole strip. The lengths of the dipole - arm strips and the microstrip balun are approximately a quarter-wavelength [4-6]. Based on matching techniques theory, the integrated balun affects the current flow at each dipole - arm, because of cancellation of the current flow to ground on the outside part of the outer conductor in corresponding coaxial line. In fact, the balun configuration ensures that the currents which flow the dipole arms become quite identical without eliminating the radiation efficiency [6].

The structure parameters of the proposed dipole for 2.4 GHz frequency point are listed as follows: 
Dipole strips: length $\mathrm{L}_{1}=20.8 \mathrm{~mm}$, width $\mathrm{W}_{1}=6 \mathrm{~mm}$, dipole gap: $\mathrm{g}_{1}=3 \mathrm{~mm}$;

Microstrip balun: length $\mathrm{L}_{2}=32 \mathrm{~mm}, \mathrm{~L}_{3}=16 \mathrm{~mm}$, $\mathrm{L}_{4}=3 \mathrm{~mm}, \mathrm{~L}_{5}=3 \mathrm{~mm}$, width $\mathrm{W}_{2}=3 \mathrm{~mm}, \mathrm{~W}_{3}=5 \mathrm{~mm}$, $\mathrm{W}_{4}=3 \mathrm{~mm}$, gap $\mathrm{g}_{2}=1 \mathrm{~mm}$;

Via radius: $r=0.375 \mathrm{~mm}$;

Ground plane: length $\mathrm{L}_{6}=12 \mathrm{~mm}$, width $\mathrm{W}_{5}=17$ $\mathrm{mm}$;

Side of microstrip bend: $l$ variable;

Side of dipole strip bend: $w$ variable.

Accurate dimensions of each part of dipole and integrated balun have numerically been computed and investigated $[4,5,7]$. These parameters were specified to achieve desired performance of the printed dipole antenna in the frequency of $2.4 \mathrm{GHz}$. Both simulated and experimental results introduce this performance. The prototype printed dipole antenna is shown in Figure 2.

This prototype dipole on which the discontinuities are not investigated ( $l$ and $w$ unchanged) has frequency resonance at about $2.45 \mathrm{GHz}$ and an efficient bandwidth for wireless applications at $2.4 \mathrm{GHz}$ - ISM band.

An interesting approximation for these discontinuities
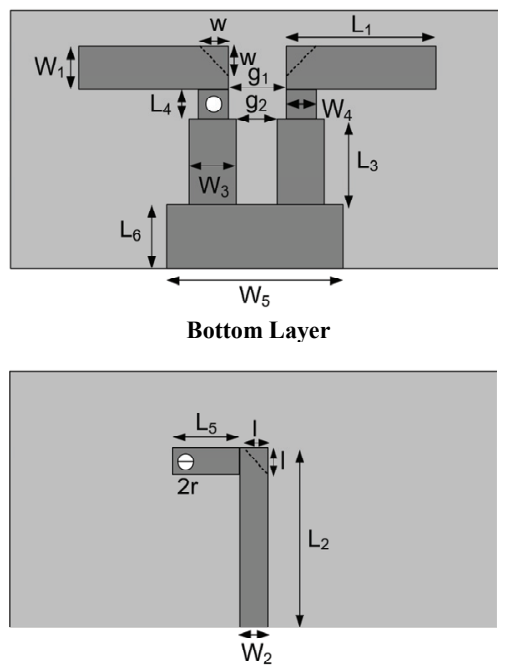

Top Layer

Figure 1. Geometry of printed dipole antenna with integrated microstrip balun.

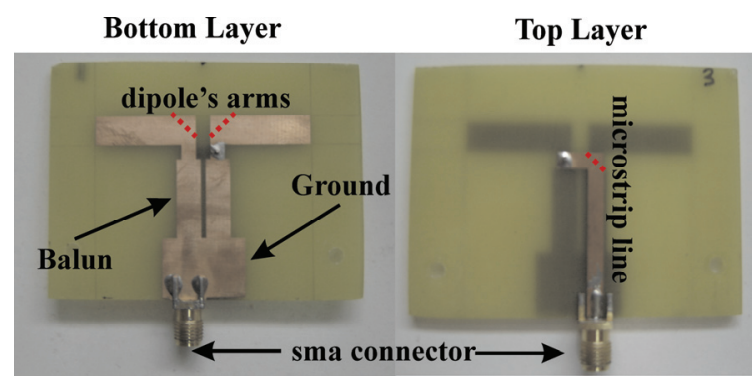

Figure 2. Prototype printed dipole. is proposed. As already mentioned, the right - angle bends at the microstrip feed line and at the strip arms of the printed dipole are inevitable discontinuities and can cause degradation in circuit performance. This is due to the fact that such discontinuities introduce parasitic reactances which can lead to phase and amplitude errors, input and output mismatch and possibly spurious coupling [8]. Based on discontinuities' forms, microwave engineering theory has proposed thoughts to face the difficulties. One approach in order to eliminate this effect is to compensate the discontinuity directly, by chamfering or mitering the conductor. That way, the excess capacitance at the bend is reduced [8]. It is generally known that the optimum value of the miter length depends on the characteristic impedance and the bend angle. This is the purpose of the proposed procedure. The variation's impact of the corresponding parameters 1 and $\mathrm{w}$ on return loss, resonance bandwidth and radiation pattern have been investigated. About resonance bandwidth definition is specified as the frequency range in which return loss is less than $-10 \mathrm{~dB}$.

These variations on the values of the 1 and $\mathrm{w}$ parameters introduce geometrical modifications in the prototype dipole architecture so as to obtain the corresponding different printed dipole antennas. Therefore, based on the prototype antenna design (Figure 2) sixteen different printed dipole antennas have been designed and implemented. Each of them has different value of $l$ and $w$ parameter. Both these values are ranged from $0 \mathrm{~mm}$ to 3 $\mathrm{mm}$, respectively. The return loss and radiation pattern of the corresponding printed dipole antenna in each case were investigated.

\section{Results and Discussion}

The defined variations of $l$ and $w$ parameters in the prototype printed dipole cause an interesting amount of simulated and measured results. More precisely, the return loss measurements are presented in four groups of printed dipole antennas. Each of them corresponds to four dipoles that have the same value of $\mathrm{w}$ parameter, but also have different value of $l$ parameter, ranging from 0 $\mathrm{mm}$ to $3 \mathrm{~mm}$. The corresponding simulated results are presented in Figures 3, 4, 5 and 6 respectively.

These curves provide that the return loss characteristics of the printed dipole are affected only by the value of 1 parameter. In fact, the resonance of them is independent of the value of $l$ and $w$ parameters. Moreover, in the frequency range of the resonance bandwidth the form of the curve becomes quite more flat as the value of $l$ parameter increases. On the other hand, the length of $w$ geometrical parameter does not affect the form of these curves. These observations are also indicated by the corresponding experimental results. 


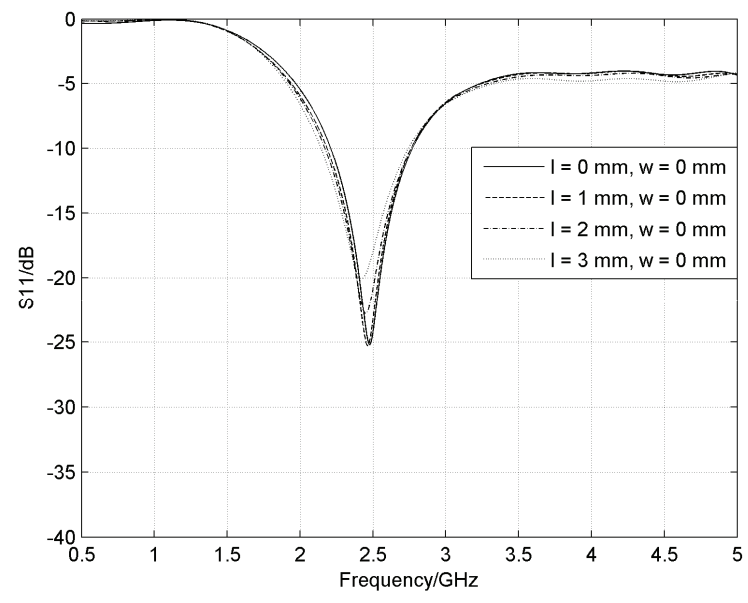

Figure 3. Simulated return loss of the printed dipole antenna for $4 l$ parameter values and for $w$ parameter equals to 0 mm.

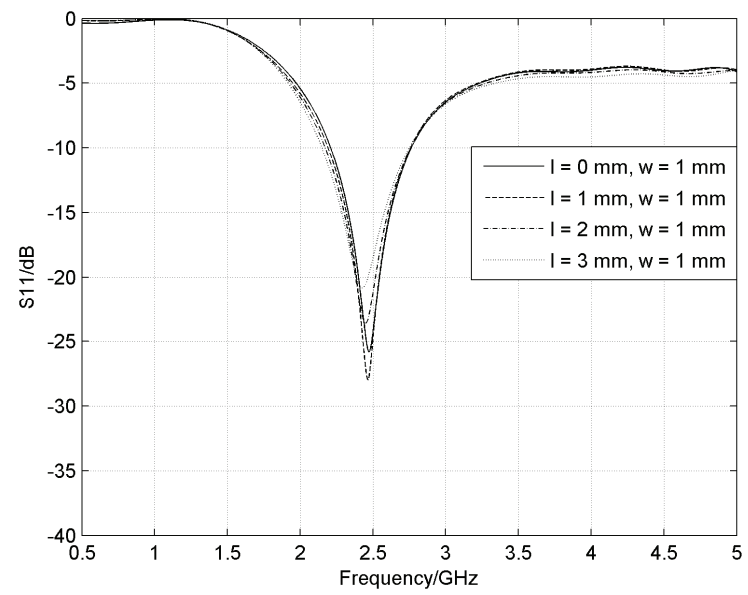

Figure 4. Simulated return loss of the printed dipole antenna for $4 l$ parameter values and for $w$ parameter equals to $1 \mathrm{~mm}$.

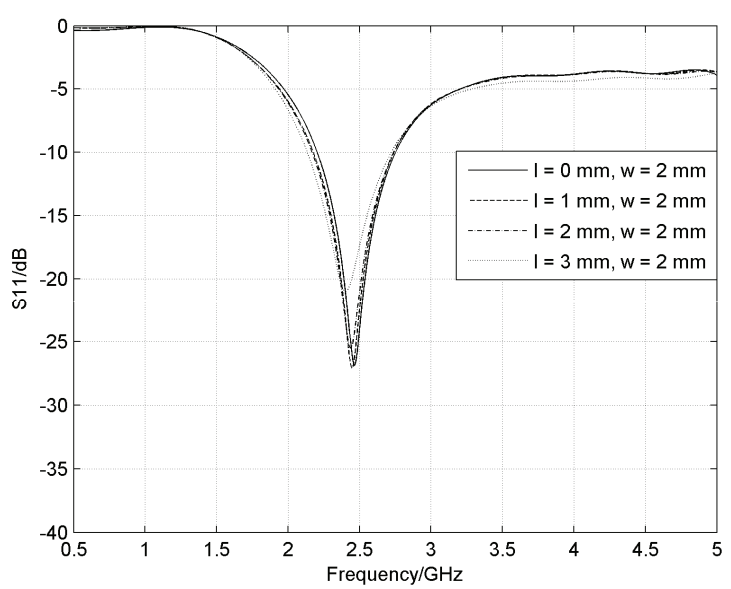

Figure 5. Simulated return loss of the printed dipole antenna for $4 \boldsymbol{l}$ parameter values and for $w$ parameter equals to 2 mm.
These are provided by a Network Analyzer and are also presented in Figures 7, 8, 9 and 10, respectively.

From these curves a relatively good agreement between the simulated and measured return loss was observed. For each value of $l$ and $w$ parameters the measured frequency point of minimum return loss is approximately $2.45 \mathrm{GHz}$ and the corresponding resonance bandwidth ranges from $2.20 \mathrm{GHz}$ to $2.75 \mathrm{GHz}$. Another issue is that the experimental results of return loss presents a resonance point on frequency range of $4 \mathrm{GHz}$. This result is not in good agreement with the simulation results. This difference may be due to effects of coaxial-to-microstrip transition, included in the measurements but not taken into account in the simulated results. In addition, the impact of 1 and $\mathrm{w}$ parameters seems not to have important interest in the frequency range of $4 \mathrm{GHz}$.

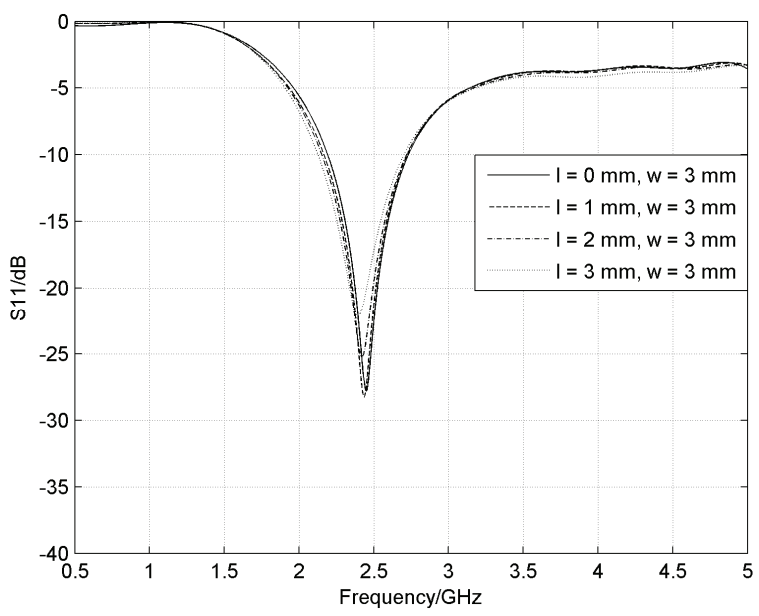

Figure 6. Simulated return loss of the printed dipole antenna for $4 \boldsymbol{l}$ parameter values and for $w$ parameter equals to $3 \mathbf{~ m m}$.

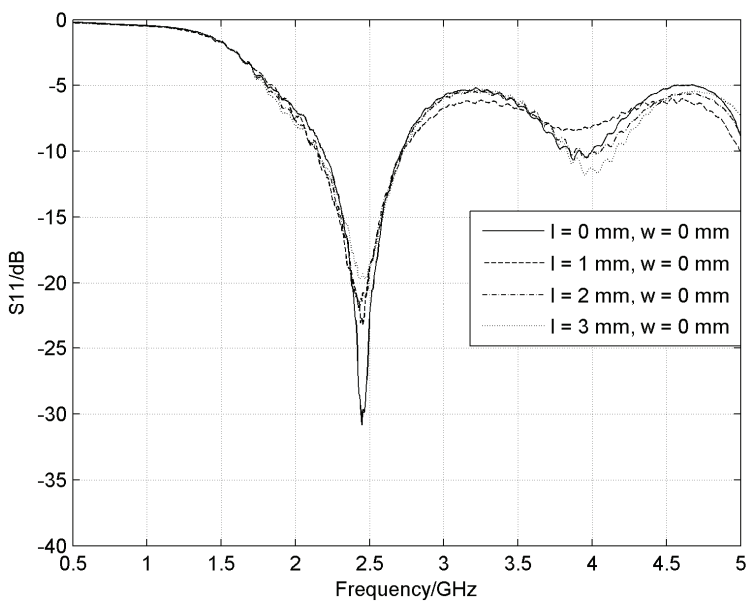

Figure 7. Measured return loss of the printed dipole antenna for $4 l$ parameter values and for $w$ parameter equals to $0 \mathrm{~mm}$. 


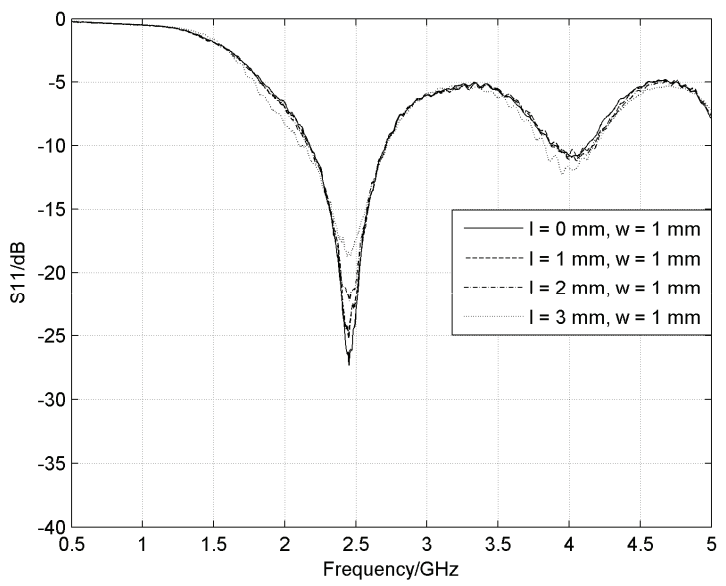

Figure 8. Measured return loss of thfde printed dipole antenna for $4 \boldsymbol{l}$ parameter values and for $\boldsymbol{w}$ parameter equals to $1 \mathrm{~mm}$.

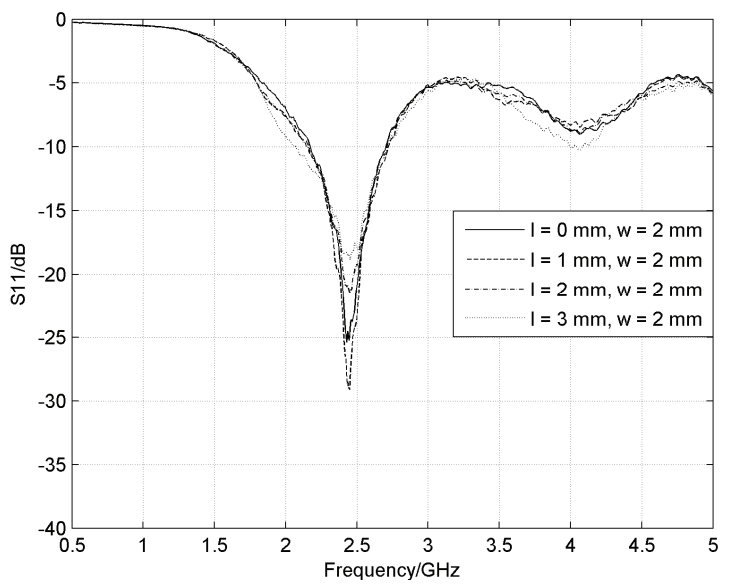

Figure 9. Measured return loss of the printed dipole antenna for $4 l$ parameter values and for $w$ parameter equals to $2 \mathrm{~mm}$.

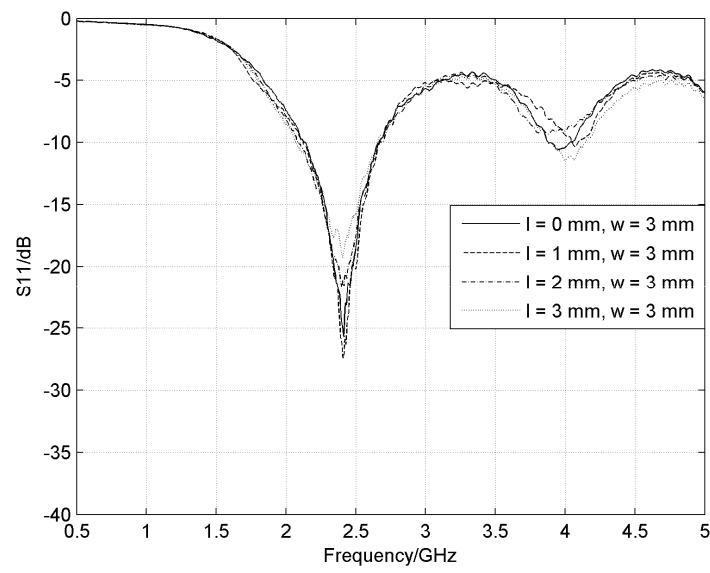

Figure 10. Measured return loss of the printed dipole antenna for $4 \boldsymbol{l}$ parameter values and for $\boldsymbol{w}$ parameter equals to $3 \mathbf{~ m m}$.
On the other hand, both simulated and experimental return loss results indicate that as the value of 1 parameter increases, the shape of the corresponding curve becomes flat for wider frequency range but the frequency point of minimum remains quite stable, simultaneously. This observations shows that return loss may be demonstrated for a specific frequency range as parameter $l$ is adjusted. Therefore, for better operation of printed dipole antenna at frequency point of interest, some of its geometric characteristics have to be modified. Both simulated and measured results indicate that a value of $l$ parameter may be considered to be important to achieve efficient return loss measurements for the printed dipole antenna design. Instead, this observation for the w parameter does not exist. Return loss and resonance bandwidth seem to be independent of $w$ parameter variation. Figures 11 and 12 present the simulated and experimental results of return loss for $l=0 \mathrm{~mm}$ and $\mathrm{w}$ variation from $0 \mathrm{~mm}$ to $3 \mathrm{~mm}$ with step $1 \mathrm{~mm}$. These curves confirm the corresponding independence.

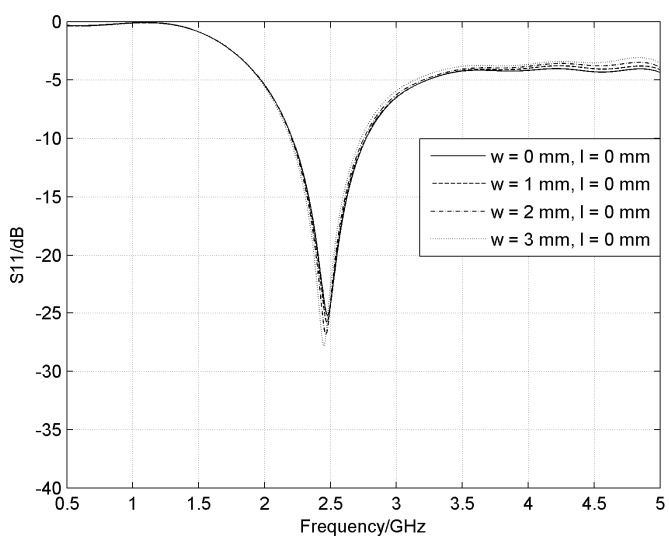

Figure 11. Simulated return loss of the printed dipole antenna for $4 w$ parameter values and for $\boldsymbol{l}$ parameter equals to $0 \mathrm{~mm}$.

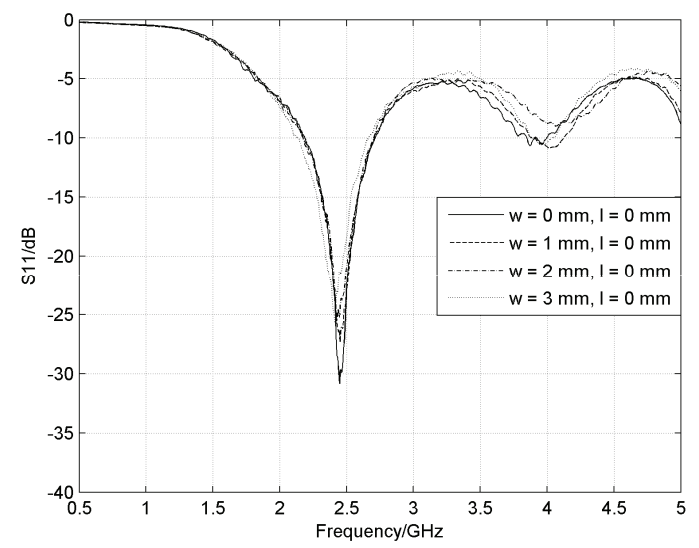

Figure 12. Measured return loss of the printed dipole antenna for $4 w$ parameter values and for $\boldsymbol{l}$ parameter equals to 0 mm. 
Based on these criteria, the proposed investigation of $l$ and $w$ parameter offers a way to implement printed dipole antenna elements with the same geometric characteristics and without declinations between them on the return loss characteristics. This observation provides an attractive solution on antenna array implementations at modern wireless applications which are constituted by a specific number of usually same antenna elements. In fact, it is observed that as the resonance bandwidth becomes more flat and uniform, the agreement between the corresponding return loss figures of geometrically identical dipoles become more practicable and realized. From this, it is also provided that the $l$ parameter is proposed to approximate the value of $2 \mathrm{~mm}$, as an optimum value for better performance. These observations are also reinforced by the unchanged frequency range of the resonance bandwidth in each case.

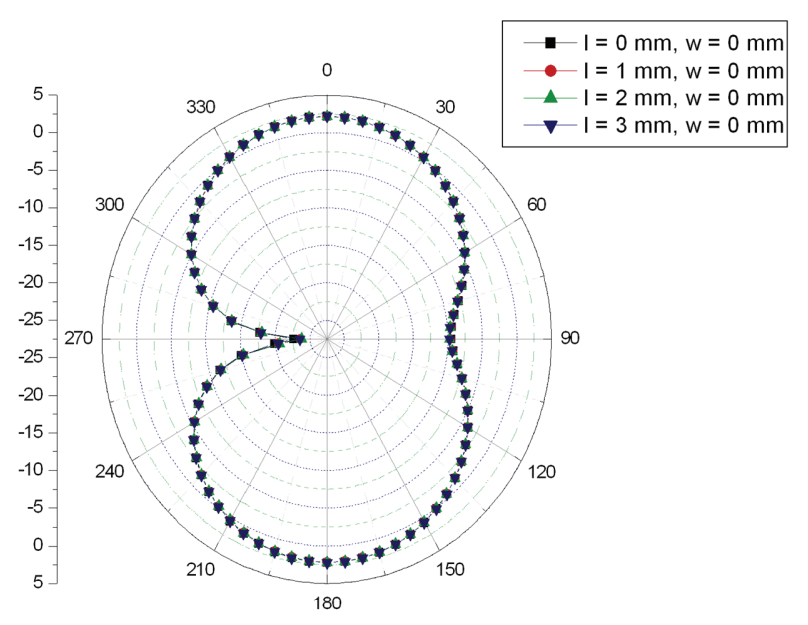

(a)

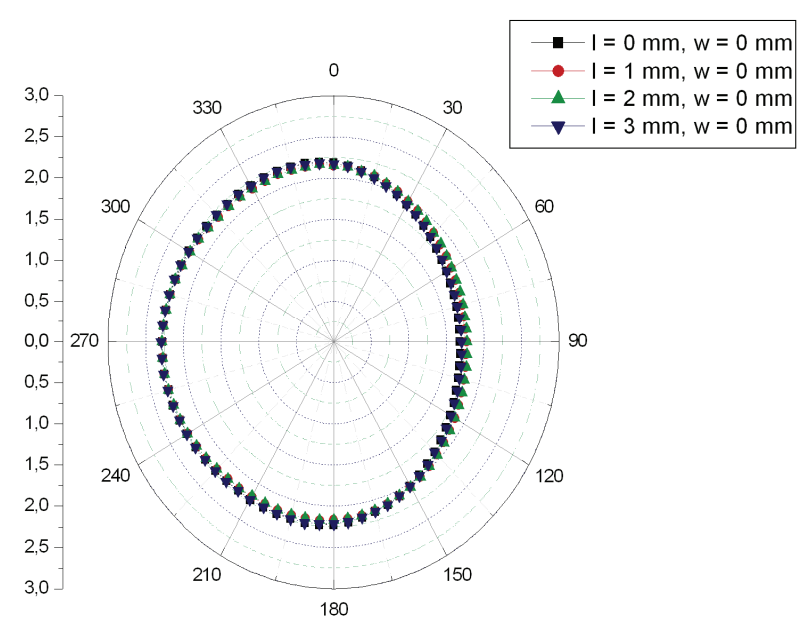

(b)

Figure 13. Simulation: Printed dipole antenna radiation patterns for $4 l$ parameter values and for $w$ parameter equals to $0 \mathrm{~mm}$, (a) $\mathrm{E}$ - plane, (b) $\mathrm{H}$ - plane.
Besides, the radiation patterns of printed dipole antennas as $l$ and $w$ parameters vary are also presented. Figures 13, 14, 15 and 16 show these radiation diagrams

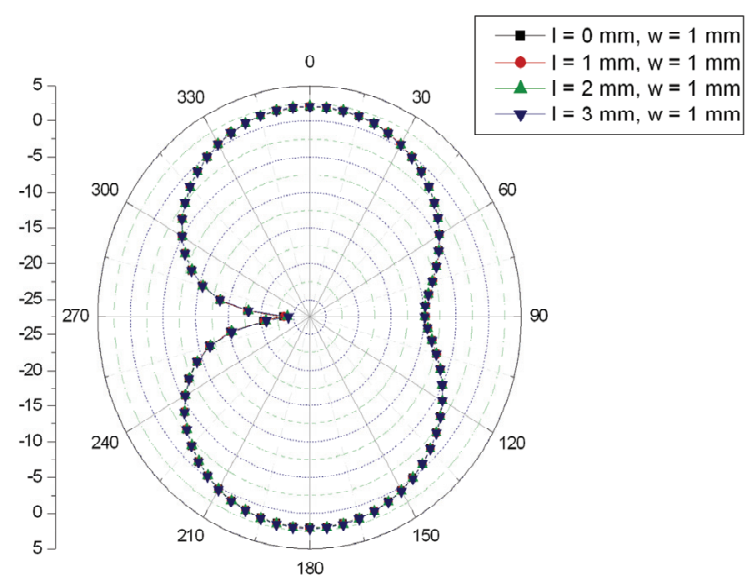

(a)

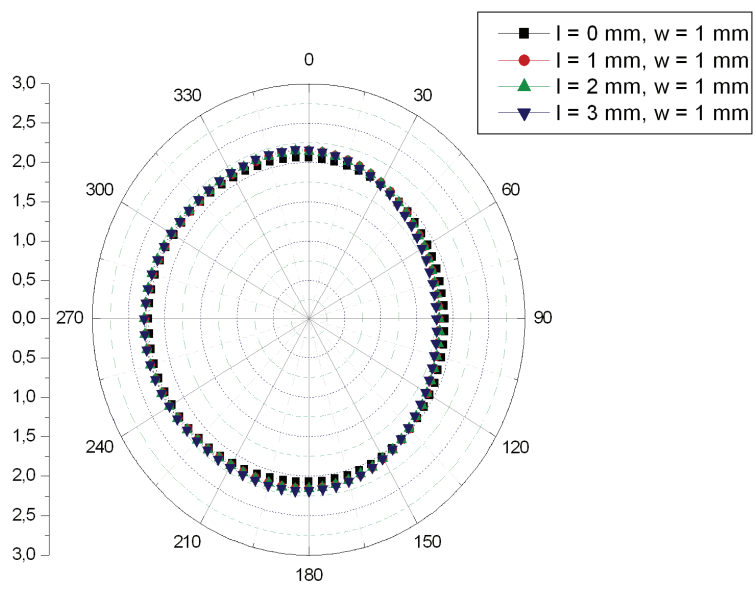

(b)

Figure 14. Simulation: Printed dipole antenna radiation patterns for $4 l$ parameter values and for $w$ parameter equals to $1 \mathrm{~mm}$, (a) $\mathrm{E}$ - plane, (b) $\mathrm{H}$ - plane.

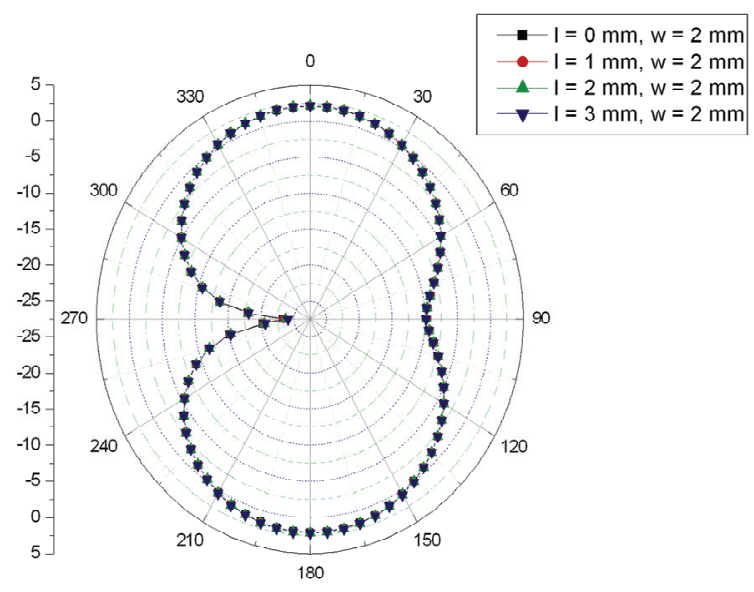

(a) 


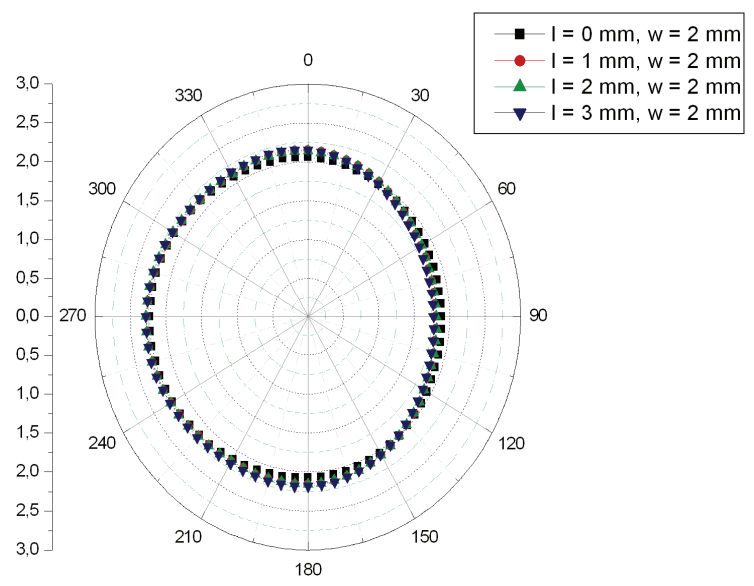

(b)

Figure 15. Simulation: Printed dipole antenna radiation patterns for $4 l$ parameter values and for $w$ parameter equals to $2 \mathrm{~mm}$, (a) $\mathrm{E}$ - plane, (b) $\mathrm{H}$ - plane.

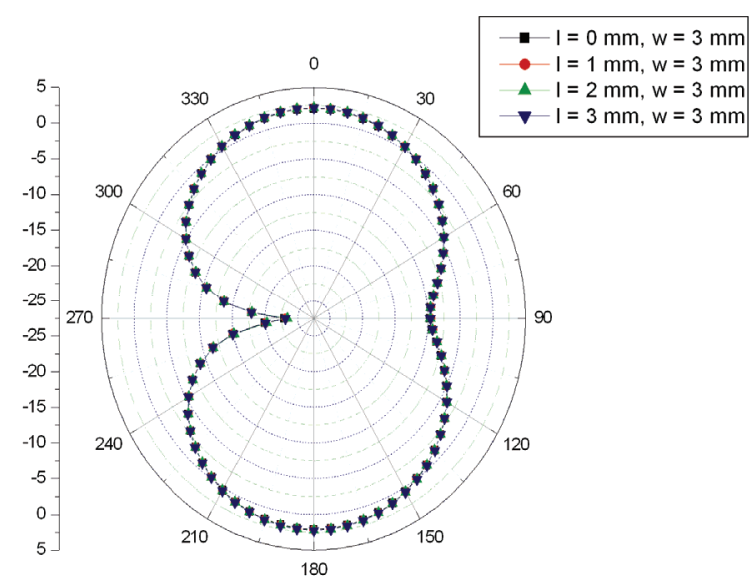

(a)

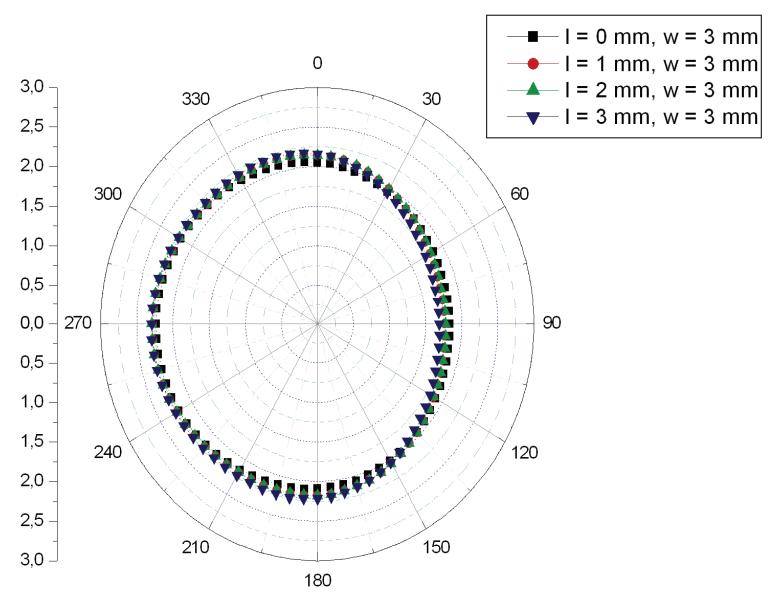

(b)

Figure 16. Simulation: Printed dipole antenna radiation patterns for $4 \boldsymbol{l}$ parameter values and for $w$ parameter equals to $3 \mathrm{~mm}$, (a) $\mathbf{E}$ - plane, (b) $\mathrm{H}$ - plane. at $2.45 \mathrm{GHz}$. Each of them corresponds to a defined value of $\mathrm{w}$ parameter and four different values of $l$ parameter, too. From these figures, the corresponding results do not indicate differences as the radiation element remains the same ( $w=$ constant), so the values of $l$ and $w$ parameters do not affect the radiation characteristics of printed dipole antenna.

\section{Conclusions}

A printed dipole antenna with integrated balun is studied and investigated. The complete structure has implemented, simulated and experimentally measured for several values of $l$ and $w$ parameters. Good agreement between simulated and measured results on return loss and resonance bandwidth has been achieved. Simulated radiation pattern has also been specified for each value of 1 and $\mathrm{w}$ parameters. Return loss seems to be affected by the variation of $l$ parameter, but resonance bandwidth and radiation diagram do not depend on it. Moreover, the w parameter variation on dipole's geometry does not provide changes on reflection coefficient values and polar curves of radiation pattern. A proposed value of $l$ parameter is also specified for identical return loss characteristics among printed dipole antennas with similar geometry characteristics. In general, as the value of $l$ increases, the return loss of the antenna becomes more flat for wider frequency range. This effect is crucial for innovator wireless communication engineering and especially antenna array design.

\section{Acknowledgements}

This research project (PENED) is co-financed by E. U.European Social Fund (80\%) and the Greek Ministry of Development-GSRT (20\%).

\section{References}

[1] D. Edward and D. Rees, "A Broadband Printed Dipole with Integrated Balun," Microwave Journal, Vol. 30, No. 5, 1987, pp. 339-344.

[2] N. Michishita, H. Arai, M. Nakano, T. Satoh and T. Matsuoka, "FDTD Analysis for Printed Dipole Antenna with Balun," Asia Pacific Microwave Conference, Sydney, 2000, pp. 739-742.

[3] G. S. Hilton, C. J. Railton, G. J. Ball, A. L. Hume and M. Dean, "Finite-Difference Time-Domain Analysis of a Printed Dipole Antenna," 19th International IEEE Antennas and Propagation Conference, Eindhoven, 1995, pp. 72-75.

[4] H.-R. Chuang and L.-C. Kuo, "3-D FDTD Design Analysis of a $2.4 \mathrm{GHz}$ Polarization-Diversity Printed Dipole Antenna with Integrated Balun and Polarization- 
Switching Circuit for Wlan and Wireless Communication Application," IEEE Transactions on Microwave Theory and Techniques, Vol. 51, No. 2, 2003, pp. 374-381.

[5] C. Votis, V. Christofilakis and P. Kostarakis, "Geometry Aspects and Experimental Results of a Printed Dipole Antenna," International Journal Communications, Network and System Sciences, Vol. 3, No. 2, 2010, pp. 97100.
[6] C. A. Balanis, "Antenna Theory Analysis and Design," Wiley Interscience, New York, 1997

[7] R. Garg, P. Bhartia, I. Bahl and A. Ittipiboon, "Microstrip Antenna Design Handbook," Artec House, Canton, 2001.

[8] D. M. Pozar, "Microwave Engineering," Wiley, New York, 1998. 